\title{
Étude de l'influence des facteurs environnementaux sur la distribution de différentes populations bactériennes dans une station mytilicole de la lagune de Bizerte (Nord-Tunisie) Effect of environmental factors on the distribution of different bacterial populations in a shellfish farming area of the lagoon of Bizerte (Northern Tunisia)
}

\author{
Imen Boukef-Ben Omrane, Monia El Bour, Salma El Mejri, Béchir Bjaoui, \\ Radhia Mraouna, Ali Harzallah et Abdellatif Boudabous
}

Volume 22, numéro 1, 2009

URI : https://id.erudit.org/iderudit/019825ar

DOI : https://doi.org/10.7202/019825ar

Aller au sommaire du numéro

Éditeur(s)

Université du Québec - INRS-Eau, Terre et Environnement (INRS-ETE)

ISSN

0992-7158 (imprimé)

1718-8598 (numérique)

Découvrir la revue

Citer cet article

Omrane, I. B., Bour, M. E., Mejri, S. E., Bjaoui, B., Mraouna, R., Harzallah, A. \& Boudabous, A. (2009). Étude de l'influence des facteurs environnementaux sur la distribution de différentes populations bactériennes dans une station mytilicole de la lagune de Bizerte (Nord-Tunisie). Revue des sciences de l'eau / Journal of Water Science, 22(1), 79-91. https://doi.org/10.7202/019825ar
Résumé de l'article

La présente étude a été effectuée en vue d'évaluer l'effet des fluctuations des paramètres abiotiques sur la distribution de différentes populations bactériennes viables dans la station mytilicole la plus productrice de la lagune de Bizerte (Nord-Tunisie). Le suivi a été établi pendant une année (septembre 2004 - août 2005) au niveau des neuf tables du site.

Les dénombrements bactériens des coliformes totaux (CT), des entérocoques fécaux (EF), des Vibrionaceaes (VB) et des bactéries hétérotrophes cultivables (BHC) ont été effectués pour l'eau de surface, les moules (Mytilus galloprovincialis) ainsi que pour les sédiments. Ces charges bactériennes ont été corrélées à la température de l'eau, l'oxygène dissous, la salinité, le $\mathrm{pH}$, la pluviométrie et l'ensoleillement, mesurés périodiquement en tout point du site. Le suivi des paramètres bactériologiques a révélé une présence assez importante de charges bactériennes pendant toute la période d'étude, notamment dans les moules et les sédiments, avec une distribution temporelle saisonnière. L'étude statistique a montré des corrélations positives, d'une part, entre les charges en polluants fécaux et la pluviométrie et, d'autre part, entre les charges en Vibrionaceaes et la salinité et la température.

Ces résultats révèlent l'effet anthropique bactérien au niveau du site mytilicole : les rejets continentaux en hiver et la remontée des bactéries autochtones en période estivale ont une influence considérable sur la mytiliculture et son devenir dans la lagune de Bizerte. 


\title{
ÉTUDE DE L'INFLUENCE DES FACTEURS
}

ENVIRONNEMENTAUX SUR LA DISTRIBUTION DE

DIFFÉRENTES POPULATIONS BACTÉRIENNES

DANS UNE STATION MYTILICOLE

DE LA LAGUNE DE BIZERTE (NORD-TUNISIE)

\author{
Effect of environmental factors on the distribution of different bacterial populations \\ in a shellfish farming area of the lagoon of Bizerte (Northern Tunisia)
}

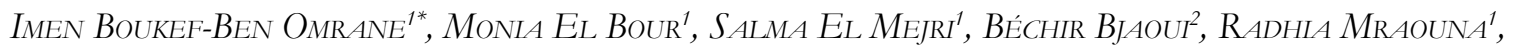
Ali Harzallah'2, AbDELlatif Boudabous ${ }^{3}$

${ }^{1}$ Laboratoire de Microbiologie, Institut National des Sciences et Technologies de la Mer, Rue 18 Mars 1934 - 2025 Salammbô, Tunisie.

${ }^{2}$ Laboratoire du Milieu Marin, Institut National des Sciences et Technologies de la Mer, Rue 18 Mars 1934 - 2025 Salammbô, Tunisie.

${ }^{3}$ Laboratoire des Microorganismes et Biomolécules Actives, Faculté des Sciences de Tunis, El Manar II, Tunisie

Reçu le 11 juin 2007, accepté le 23 mai 2008

\section{RÉSUMÉ}

La présente étude a été effectuée en vue d'évaluer l'effet des fluctuations des paramètres abiotiques sur la distribution de différentes populations bactériennes viables dans la station mytilicole la plus productrice de la lagune de Bizerte (Nord-Tunisie). Le suivi a été établi pendant une année (septembre 2004 - août 2005) au niveau des neuf tables du site.

Les dénombrements bactériens des coliformes totaux (CT), des entérocoques fécaux (EF), des Vibrionaceaes (VB) et des bactéries hétérotrophes cultivables (BHC) ont été effectués pour l'eau de surface, les moules (Mytilus galloprovincialis) ainsi que pour les sédiments. Ces charges bactériennes ont été corrélées à la température de l'eau, l'oxygène dissous, la salinité, le $\mathrm{pH}$, la pluviométrie et l'ensoleillement, mesurés périodiquement en tout point du site.

Le suivi des paramètres bactériologiques a révélé une présence assez importante de charges bactériennes pendant toute la période d'étude, notamment dans les moules et les sédiments, avec une distribution temporelle saisonnière. Létude statistique a montré des corrélations positives, d'une part, entre les charges en polluants fécaux et la pluviométrie et, d'autre part, entre les charges en Vibrionaceaes et la salinité et la température.

Ces résultats révèlent l'effet anthropique bactérien au niveau du site mytilicole : les rejets continentaux en hiver et la remontée des bactéries autochtones en période estivale ont une

*Auteur pour correspondance

Téléphone : (216) 71730420 / 97525632

Télécopieur : (216) 71732622

Courriel : imen.boukef@yahoo.fr 
influence considérable sur la mytiliculture et son devenir dans la lagune de Bizerte.

Mots clés : écosystème lagunaire, station mytilicole, bactéries hétérotrophes cultivables, coliformes totaux, entérocoques fécaux, Vibrionaceaes.

\section{ABSTRACT}

The present study aimed to assess the influence of abiotic parameters on the distribution of various viable bacterial populations in the most productive mussel farming area in the Bizerte Lagoon (Northern Tunisia). A monitoring survey was carried out over one year (September 2004 - August 2005), within all the farming tables at the station. Bacterial enumerations of total coliforms (CT), fecal enterococci (EF), Vibrionaceae (VB) and cultivable heterotrophic bacteria (BHC) were carried out for surface water, mussels (Mytilus galloprovincialis) and sediments. These bacterial counts were correlated with water temperature, dissolved oxygen, salinity, $\mathrm{pH}$, rainfall and sunshine, which were periodically measured at all points within the sampling area.

The survey of the bacteriological parameters revealed high bacterial counts during the whole monitoring period, especially in the mussels and sediments, as well as seasonal variability. Statistical analyses revealed positive correlation between the loads of fecal pollutants and rainfall during the winter period, and between the loads of Vibrionaceae and salinity and temperature during the summer period.

These results highlight the negative anthropogenic bacterial effect within the mussel farming areas: sewage discharges in winter, and the growth of autochthonous pathogenic bacteria during the summer period, have a considerable influence on the mussel culture in the Bizerte Lagoon.

Keywords: Lagoon, Mussel farming area, Culturable heterotrophic bacteria, Total coliform bacteria, Faecal enterococci, Vibrionaceae.

\section{INTRODUCTION}

En Méditerranée, la mytiliculture est, depuis la dernière décennie, sous l'influence néfaste de pression anthropique côtière de plus en plus grandissante (BABARRO et ZWAAN, 2002; CAVALLO et STABILI, 2002; CHARMASSON et al., 1999; DELLALI et al., 2001;
DRIDI et al., 2007; KHESSIBA et al., 2005; LA ROSA et al., 2001; LIZARRAGA-PARTIDA et CARDENAS, 1996; PAMPANIN et al., 2005; STABILI et al., 2005; WAECHTER et al., 2002). Il a été souvent mentionné que ce sont les charges bactériennes notamment allochtones induites par les apports anthropiques qui présentent un risque menaçant aussi bien pour la santé humaine que celle des organismes sédentaires, notamment les bivalves (CHINA et al., 2003; GOURMELON et al., 2006).

L'effet négatif de certaines bactéries allochtones induisant la mortalité des bivalves (huitres, moules et palourdes) a été décrit par YAM et al. (1999). Aussi, l'étude de WAECHTER et al. (2002) a révélé le rôle implicite de Vibrio splendidus dans des cas de mortalités de naissains d'huître méditerranéenne (Crassostrea gigas), alors que BABARRO et ZWAN (2002) ont démontré l'effet de l'anoxie, induite par le développement d'une flore bactérienne anaérobique, sur la mortalité des Mytilus edulis aux Pays-Bas.

Selon les études récentes de CUSSON et al. (2005) et RIISGARD et al. (2003), la physiologie de filtration et de rétention bactérienne chez les bivalves dans leur milieu serait sous l'influence des conditions abiotiques; ainsi, des corrélations significatives ont été établies entre les charges de bactéries dans l'eau, d'une part, et celles accumulées dans les moules, d'autre part. Ces dernières refléteraient bien l'état de leur milieu environnant (BLANCHET et al., 2003; STABILI et al., 2005; YAM et al., 1999).

En Tunisie, la mytiliculture est exclusivement pratiquée dans la lagune de Bizerte. Des essais de son introduction dans d'autres sites côtiers ont échoué (MEDHIOUB, 1990). Durant la dernière décennie, cette activité dans la lagune a connu des chutes alarmantes quant à la pérennité du bivalve (BOUAMAMA, 2001; DELLALI et al., 2000, 2001; EL BOUR et al., 2001). Ces travaux ont souligné la présence fluctuante de différentes charges bactériennes chez la moule Mytilus galloprovincialis à différentes périodes de l'année. Aussi, différents cas de mortalité de la moule dans les différents sites mytilicoles ont été signalés, notamment en périodes estivales marquées par une hausse notable en température des eaux et une oxygénation appauvrie (El HILI, 2005). Par ailleurs, l'étude de BEN MAHMOUD (2003) a révélé l'influence négative de quelques espèces bactériennes sur la reproduction de la moule dans la lagune de Bizerte. Cependant, les résultats de ces études restent sporadiques et non concluants quant à l'effet des paramètres du milieu sur la variation en charges bactériennes dans les sites mytilicoles.

En dépit d'une pollution côtière notable a proximité de la zone mytilicole la plus productrice des fermes aquacoles de la lagune de Bizerte, peu d'études se sont intéressées au 
suivi des paramètres bactériologiques dans ce site (DELLALI et al., 2000, 2001; ESSID et al., 2007). Ces études ont été limitées par un nombre réduit des stations étudiées ou par une courte durée de suivi. L'objectif de ce travail est d'étudier la distribution temporelle de différentes populations bactériennes dans l'eau, les moules et les sédiments dans ce site mytilicole, au cours d'un cycle annuel, et d'établir d'éventuelles relations entre les fluctuations des charges bactériennes et les paramètres abiotiques du milieu, ce qui permettra d'appréhender la période critique de nuisance sur la moule Mytilus galloprovincialis et son recrutement dans la lagune.

\section{MATÉRIEL ET MÉTHODES}

\subsection{Site d'étude et échantillonnage}

Le site d'étude est situé au nord-ouest de la lagune de Bizerte $\left(37^{\circ} 12^{\prime} 14^{\prime \prime} \mathrm{N}, 9^{\circ} 55^{\prime} 79^{\prime \prime} \mathrm{E}\right)$ (Figure 1). Au niveau de la station, les prélèvements ont été effectués dans les neuf tables d'élevage de la moule Mytilus galloprovincialis.

Les tables, de $2 \mathrm{~m}$ de long chacune, sont situées à une distance de 500 à 1200 m de la ligne de côte, pour une profondeur moyenne

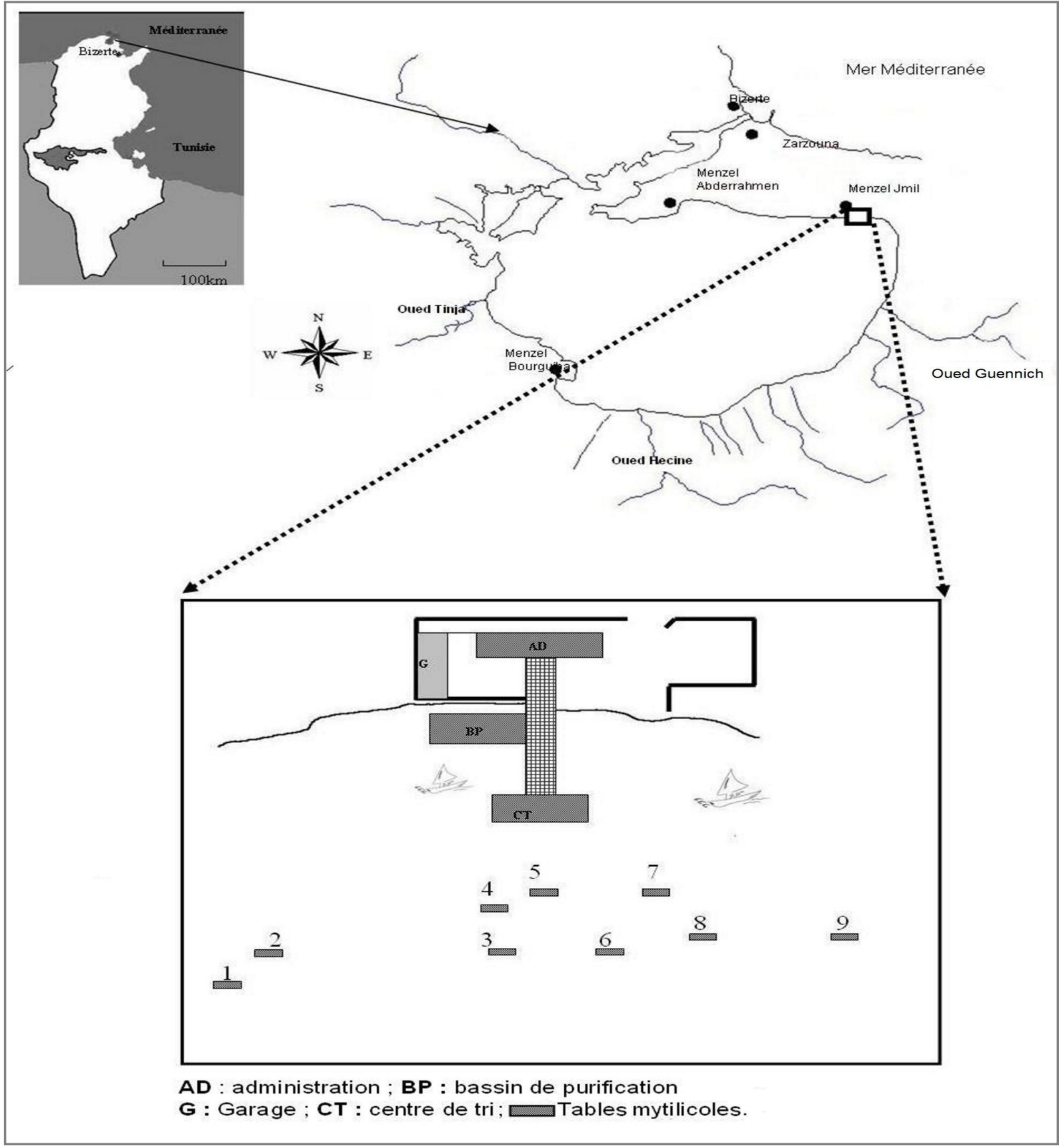

Figure 1. Le site d'étude (station mytilicole dans la région nord-est de la lagune de Bizerte) : points de prélèvements ou tables mytilicoles.

The study area (mussel farming in the north-eastern part of the Bizerte Lagoon): sampling points. 
de 4 à $8 \mathrm{~m}$. Dans chaque table, les moules sont suspendues en perches par des cordes de $3 \mathrm{~m}$ et tendues jusqu'à $0,5 \mathrm{~m}$ du fond (on compte une centaine de cordes par table).

Au niveau de chaque table, des prélèvements mensuels (durant la période de septembre 2004 jusqu'en août 2005) ont été effectués au niveau de l'eau de surface, des moules et des sédiments (dans la zone en dessous de la table).

Les échantillons ont été effectués le matin et à la même heure. Chaque prélèvement de moules consiste en un lot de dix individus vivants par table. Les prélèvements d'eaux ont été effectués dans des flacons en verre (Pyrex) stériles de $500 \mathrm{~mL}$ (à $50 \mathrm{~cm}$ sous la surface d'eau). Les prélèvements de sédiments ont été effectués à l'aide d'une benne manuelle sous chaque table puis conservés dans des sacs en plastique stériles. Tous les échantillons effectués sont maintenus à $4{ }^{\circ} \mathrm{C}$ et analysés dans les 24 heures qui suivent les prélèvements.

\subsection{Paramètres abiotiques}

Ces paramètres sont la température de l'eau, l'oxygène dissous, la salinité et le $\mathrm{pH}$. Tous ces paramètres ont été mesurés mensuellement in situ à l'aide d'une sonde multiparamétrique (type WTW) au niveau des différents points de prélèvements ou tables mytilicoles. Les relevés des moyennes mensuelles de pluviométrie $(\mathrm{mm})$, ainsi que les durées d'ensoleillement (en heures) ont été enregistrés par une station météorologique située à $10 \mathrm{~km}$ de la station mytilicole.

\subsection{Analyses bactériologiques}

Les prélèvements d'eau ont été analysés à l'état brut ou dilués dans l'eau physiologique à $3 \%$ de $\mathrm{NaCl}$. Pour les moules, au préalable de l'analyse, les individus (un lot de dix moules) sont brossés puis écaillés aseptiquement, leurs contenus (chaire et liquide intravalvaire) sont vidés dans un sac stérile et homogénéisés dans l'eau physiologique à $3 \% \mathrm{de} \mathrm{NaCl}(\mathrm{w} / \mathrm{v})$ à l'aide d'un Stomacher (STOMACHER 400, Seward). Pour le sédiment, $10 \mathrm{~g}$ de prise d'essai sont mis en suspension dans un flacon stérile contenant de l'eau physiologique à $3 \% \mathrm{de} \mathrm{NaCl}$ $(w / v)$. L'homogénat est analysé à l'état brut ou dilué.

Les différents types de dénombrements ont été effectués selon la technique de comptage en boîtes gélosées ou «Plate Counting Agar» (PCA) (ISO 15214, 1998) se basant sur l'utilisation de milieux sélectifs : Désoxycolate Lactosé (DL), Slanetz and Brathely (SB), Thiosulfate-Citrate-Bile-Sodium (TCBS) et Zobell ( $3 \%$ de $\mathrm{NaCl}$ ), respectivement pour les dénombrements des coliformes totaux (CT), entérocoques fécaux (EF), Vibrionacaes (VB) et bactéries hétérotrophes cultivables (BHC).

Pour les prélèvements d'eau, les dénombrements des polluants fécaux (coliformes et entérocoques) ont été réalisés par la technique de filtration sur membranes quadrillées de porosité $0,45 \mu \mathrm{m}$ (nitrate de cellulose), utilisant les milieux respectifs $\mathrm{DL}$ et $\mathrm{SB}$. Toutes les boîtes ensemencées sont incubées pendant 24 à 48 heures à $37^{\circ} \mathrm{C}$ avant leur lecture. À chaque dénombrement, la charge en bactéries est estimée en nombres d'unités formant colonies (ufc) par $100 \mathrm{~mL}$ d'eau ou d'homogénat de moules ou de sédiments.

\subsection{Analyses statistiques}

Les corrélations entre les différentes charges en populations bactériennes et les paramètres du milieu ont été déterminées par utilisation du test de coefficient de corrélation Pearson (r) à l'aide du logiciel de statistique SPSS 12.0. L'analyse de la composante principale (ACP) a été appliquée en utilisant le Statbox ${ }^{\mathrm{TM}}$ software (Microsoft Excel). Les valeurs brutes des dénombrements des charges bactériennes $(\mathrm{x})$ sont transformées au moyen de l'équation $\mathrm{y}=\log _{10}(\mathrm{x}+1)$.

\section{RÉSULTATS}

\subsection{Paramètres abiotiques}

Pour ces paramètres (température de l'eau, oxygène dissous, salinité et $\mathrm{pH}$ ), des moyennes mensuelles ont été déterminées pour chaque point du site étudié (au niveau des neuf tables mytilicoles) (Figure 2).

Selon les résultats obtenus, la température moyenne de l'eau est de $18{ }^{\circ} \mathrm{C}$. Cette moyenne a oscillé entre $9,2^{\circ} \mathrm{C}$ en février et $25^{\circ} \mathrm{C}$ en août, avec des variations brusques notables à l'automne et au printemps. Pour l'oxygène dissous, les teneurs moyennes ont varié entre $3,9 \mathrm{mg} \bullet \mathrm{L}^{-1}$ (juillet) et $8 \mathrm{mg} \bullet \mathrm{L}^{-1}$ (janvier), avec une oxygénation plus importante en période hivernale. Une corrélation négative entre la température et les taux en oxygène dissous a été trouvée $(\mathrm{r}=-0,689, \mathrm{p}<0,05)$. La salinité a varié entre 17,4 psu (mars) et 37,6 psu (octobre). Les eaux de la lagune sont sous influence marine (en période estivale) et continentale (apports du bassin versant en hiver). Ainsi, une faible salinité est enregistrée en fin d'hiver (février-avril). Aussi, une corrélation négative entre la salinité et la pluviométrie a été constatée $(r=-0,810, p<0,01)$. 


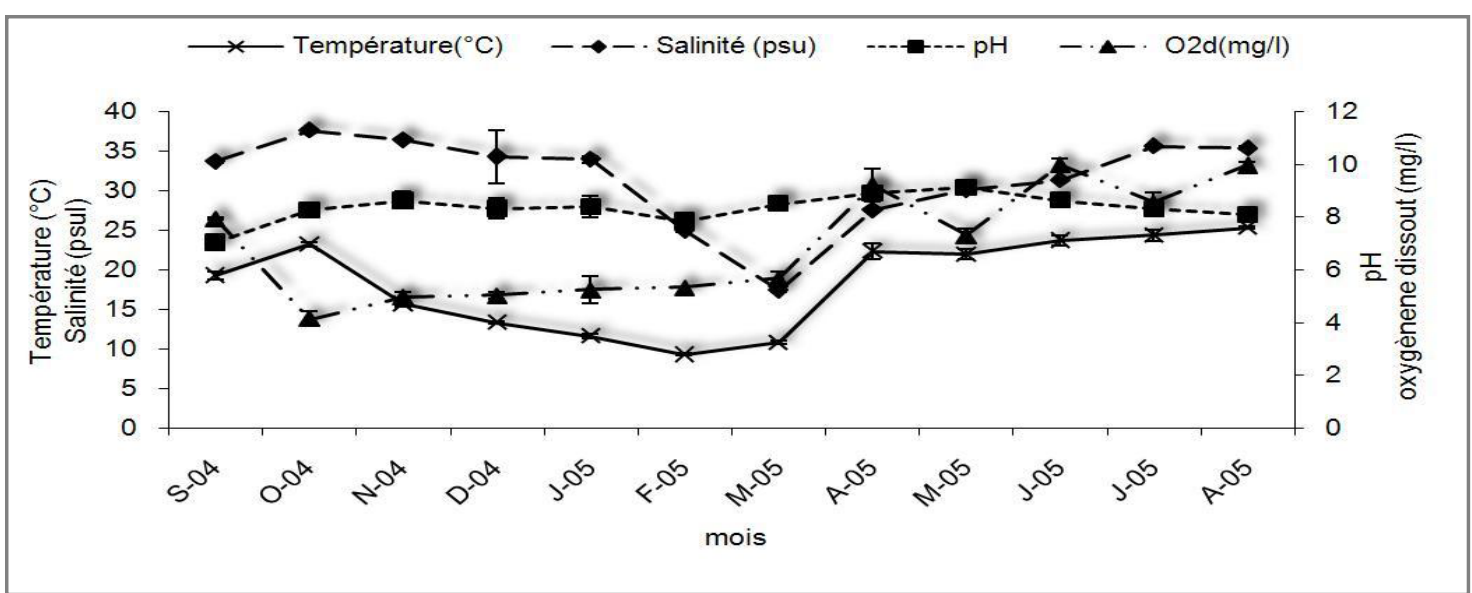

Figure 2. Évolution mensuelle des paramètres physico-chimiques dans le site d'étude : température de l'eau, oxygène, salinité et $\mathrm{pH}$.

Monthly evolution of the physico-chemical parameters in the study area: water temperature, dissolved oxygen, salinity and $\mathrm{pH}$.

Pour le $\mathrm{pH}$, les variations ne sont pas notables $(7<\mathrm{pH}<9,1)$. Ce paramètre est resté relativement stable durant la période d'étude.

Les résultats relatifs aux suivis de la pluviométrie et l'ensoleillement sont donnés par la figure 3a. Ainsi, la moyenne pluviométrique mensuelle pour toute la région lagunaire a fluctué entre $0,7 \mathrm{~mm}$ (juin) et $210 \mathrm{~mm}$ (février) avec un apport mensuel de $81 \mathrm{~mm}$. Les quantités les plus importantes ont été enregistrées en période automno-hivernale $(179 \mathrm{~mm})$. Pour l'ensoleillement, la durée moyenne mensuelle a fluctué entre 106 heures et 373 heures. Aussi, il a été montré que la période de moindre ensoleillement correspond à la période pluvieuse (novembre - février) avec une durée $<200 \mathrm{~h} \bullet \mathrm{mois}^{-1}$. Une corrélation négative entre la durée d'ensoleillement et la moyenne pluviométrique a été aussi démontrée $(\mathrm{r}=-0,905, \mathrm{p}<0,01)$. Comme il a été constaté que la durée d'ensoleillement mensuelle est significativement reliée à la température $(\mathrm{r}=0,814, \mathrm{p}<0,01)$ mais inversement corrélée à la teneur en oxygène $\operatorname{dissous}(\mathrm{r}=-0,771, \mathrm{p}<0,05)$.

\subsection{Suivi des différentes populations bactériennes}

Les différents dénombrements ont concerné les charges en bactéries hétérotrophes cultivables (BHC), les polluants fécaux notamment coliformes totaux (CT) et entérocoques fécaux (EF), ainsi que les Vibrionaceaes (VB) (Figure 3b, c et d).

\subsubsection{Estimation des bactéries hétérotrophes cultivables (BHC)}

Les résultats relatifs aux bactéries hétérotrophes cultivables (BHC) ont révélé des charges assez importantes pour les différents types de prélèvements $\left(>10^{5} \mathrm{cfu} \bullet 100 \mathrm{~mL}^{-1}\right)$. Cependant, les sédiments et les moules sont dix fois plus chargés en ces bactéries que l'eau (charges respectives : $2 \bullet 10^{7} \mathrm{cfu} \bullet 100 \mathrm{~mL}^{-1}$ et $\left.3 \cdot 10^{6} \mathrm{cfu} \cdot 100 \mathrm{~mL}^{-1}\right)$.

Le traitement statistique de ces résultats a révélé une corrélation positive entre ces charges bactériennes dans l'eau et l'apport pluviométrique $(\mathrm{r}=0,596, \mathrm{p}<0,05)$, d'une part, et avec les charges en polluants fécaux, d'autre part : pour les CT $(r=0,621, p<0,05)$ et pour les EF $(r=0,600, p<0,05)$. Cette corrélation expliquerait l'apport important en polluants fécaux par lessivage en provenance du bassin versant en période pluvieuse.

Il a été également trouvé que les charges des BHC dans le sédiment sont corrélées positivement avec celles des Vibrionacaes $(r=0,650, p<0,05)$.

\subsubsection{Estimation des polluants fécaux (CT et EF)}

Les résultats relatifs à ces indicateurs fécaux ont révélé que les sédiments et les moules sont plus chargés (moyennes annuelles 100 fois plus importantes que dans l'eau) notamment en période automnale et hivernale (Figure 3b, c et d).

Cependant, les variations saisonnières, notamment pour les entérocoques fécaux, sont peu importantes pour les différents compartiments étudiés.

En outre, les résultats relatifs aux moules ont révélé que les charges moyennes en CT sont significativement plus importantes que celles des EF, à la différence des eaux où les deux groupes de polluants ont varié d'une façon similaire, montrant deux pics, en janvier, coïncidant avec la période pluvieuse, sachant que ces deux groupes de bactéries ont été significativement corrélés $(r=0,926, p<0,01)$. 

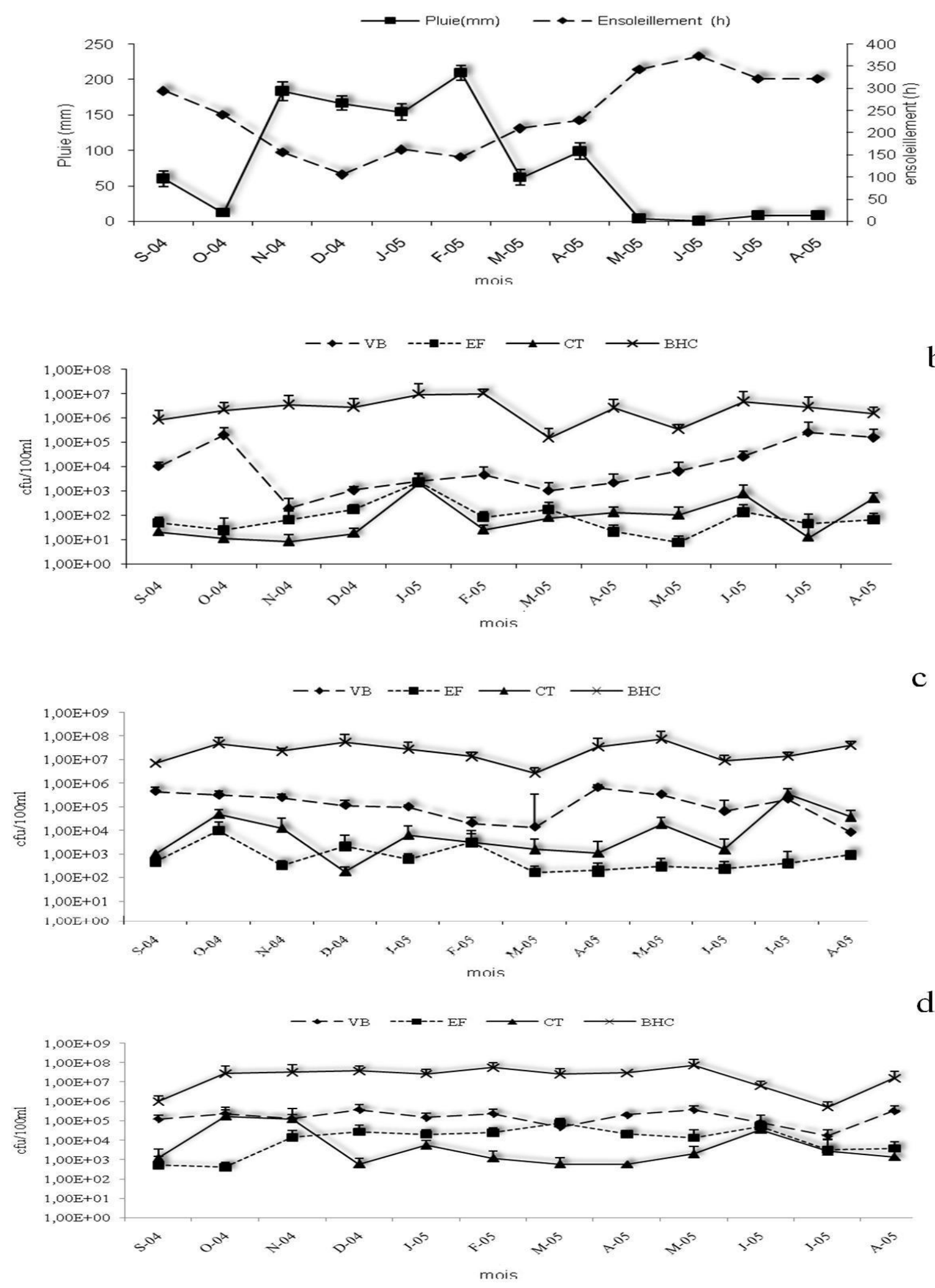

Figure 3. Variations en charges bactériennes mensuelles (cfu/100mL) dans (b) l'eau, (c) la moule et (d) le sédiment en relation aux variations en (a) facteurs abiotiques dans le site d'étude. BHC : bactéries hétérotrophes cultivables; $\mathrm{CT}$ : coliformes totaux; EF : entérocoques fécaux; VB : Vibrionaceae.

Monthly variations of the bacterial charges (cfu/ml) in (b) the water, (c) the mussel and (d) the sediment in relation with variation of (a) the abiotic factors in the study area. BHC: heterotrophic cultivable bacteria; CT: total coliforms; EF: fecal enterococci; VB: Vibrionaceae. 
D’autre part, il a été constaté que les teneurs en EF dans l'eau ont présenté une corrélation négative avec la température $(\mathrm{r}=-0,408, \mathrm{p}<0,05)$ alors que dans le sédiment, elles sont négativement corrélées avec la salinité $(\mathrm{r}=-0,775, \mathrm{p}<0,05)$ suggérant une survie moindre de ces bactéries en conditions estivales dans la colonne d'eau et les sédiments.

\subsubsection{Estimation des Vibrionaceaes (VB)}

Les résultats relatifs aux variations en $\mathrm{VB}$ au niveau des différents types de prélèvements sont illustrés sur la figure 3b, c et d. Les taux estimés ont montré que les sédiments et les moules sont les plus concentrés que l'eau notamment en saison estivale et en début d'automne.

Par ailleurs, une corrélation positive a été révélée entre la température et la charge en ces bactéries, dans les moules et l'eau (respectivement, $\mathrm{r}=0,391 \mathrm{p}<0,05$ et $\mathrm{r}=0,617$ $\mathrm{p}<0,05)$. Les taux en VB dans l'eau sont également associés avec la salinité $(r=0,483 \mathrm{p}<0,05)$. Cependant, les charges en ces bactéries dans l'eau montrent une corrélation négative avec la pluviométrie $(\mathrm{r}=-0,570, \mathrm{p}<0,05)$ à la différence des polluants fécaux, ce qui suggère une provenance du milieu (bactéries autochtones) avec prolifération favorisée par les conditions estivales.

\subsection{Analyse en composante principale}

Cette analyse a été réalisée en vue de déterminer les interrelations entre les variables bactériologiques dans la colonne d'eau et dans les moules et les paramètres du milieu considérés séparément. Se basant sur les corrélations entre les différents paramètres du milieu et les charges bactériennes (déterminées par le logiciel SPSS.12), nous avons constaté que le $\mathrm{pH}$ dont les valeurs sont presque constantes ne présente pas de corrélation avec les autres paramètres ou types bactériens, de ce fait, il ne sera pas pris en considération dans les ACP.

\subsubsection{Pour la colonne d'eau}

Ce sont les deux premiers axes (F1 et F2) qui illustrent $67,92 \%$ de la variance totale soit respectivement $51,37 \%$ et $16,55 \%$ de l'inertie (Figure 4).

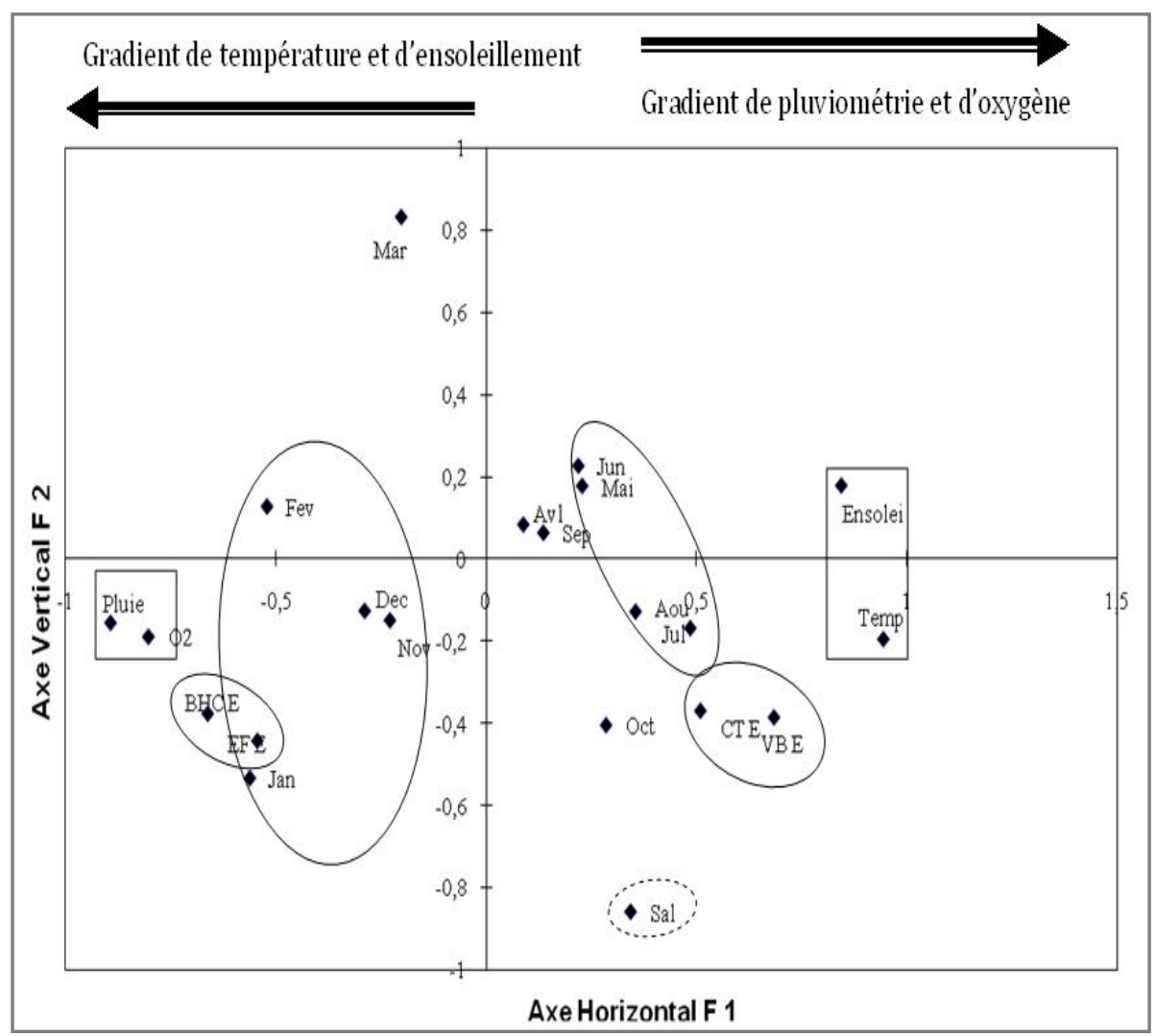

Figure 4. Analyse en composante principale des différents facteurs abiotiques, et des charges en différents types bactériens dans l'eau de surface (Temp : température; Sal : salinité; Ensolei : Ensoleillement; Pluie : Pluviométrie; $\mathrm{O}_{2}$ : taux en oxygène dissous; BHC E : bactéries hétérotrophes cultivables; CT E : coliformes totaux; EF E : entérocoques fécaux; VB E : Vibrionaceae).

Principal component analyses including the abiotic factors and charges of different bacterial populations for the surface water. (Temp: temperature; Sal: salinity; Ensolei: sunshine; Pluie: rainfall; $\mathrm{O}_{2}$ : the rate of oxygen; $\mathrm{BHC}$ E: heterotrophic cultivable bacteria; CT E: total coliforms; EF E: fecal enterococci; VB E: Vibrionaceae). 
L’axe F1 est défini sur le pôle positif par la température de l'eau et l'ensoleillement avec de fortes contributions relatives (CTR) respectives de 0,94 et 0,84. Cet axe est défini sur le pôle négatif par la pluviométrie et la teneur de l'eau en oxygène avec respectivement $\mathrm{CTR}=0,89$ et 0,8 . Sur cet axe se projettent les VB $(\mathrm{CTR}=0,68)$ et secondairement les CT $(\mathrm{CTR}=0,5)$. Aussi, ce sont les mois les plus chauds (mai, juin, juillet et août) qui se projettent sur le pôle positif défini par la température et l'ensoleillement. Ainsi, une augmentation estivale a été observée pour ces deux types de populations bactériennes (VB et des CT).

D'autre part, les BHC $(\mathrm{CTR}=0,66)$ et les EF en plus faible contribution relative $(\mathrm{CTR}=0,54)$ se projettent sur le pôle négatif de l'axe F1 défini par la pluviométrie et la teneur en oxygène dissous en même temps que les mois de novembre, décembre, janvier et février, ce qui traduirait une augmentation hivernale en ces deux types de populations bactériennes concomitantes, avec l'augmentation en pluviométrie et l'oxygénation du milieu. Par ailleurs, l'axe F2 est défini uniquement par la salinité $(\mathrm{CTR}=0,7)$ qui se projette sur le pôle négatif.

\subsubsection{Pour les moules}

Ce sont les deux premiers axes (F1 et F2) qui illustrent $62,25 \%$ de la variance totale soit respectivement $46 \%$ et $16,25 \%$ de l'inertie (Figure 5).

Dans ce cas, idem que pour l'eau, l'axe F1 est défini sur le pôle positif par la température de l'eau et l'ensoleillement avec de fortes contributions relatives respectives de CTR $=0,81$ et 0,75 , et sur le pôle négatif par la teneur des eaux en oxygène et la pluviométrie avec des CTR respectives de 0,81 de 0,74. Sur cet axe se projettent uniquement les $\mathrm{CT}$ en plus faible contribution relative $(\mathrm{CTR}=0,3)$ pour la période d'avril jusqu'en septembre qui se projettent sur le pôle positif défini par la température et l'ensoleillement. Il apparait ainsi que l'augmentation en charges bactériennes dans les moules est indépendante de la température et de l'ensoleillement.

D'autre part, les BHC $(\mathrm{CTR}=0,42)$ se projettent sur le pôle négatif de l'axe F1 défini par la pluviométrie et la teneur en oxygène dissous pour la période de novembre jusqu'en février, ce qui montre que pendant cette période, la charge bactérienne dans les moules dépend essentiellement de la pluviométrie.

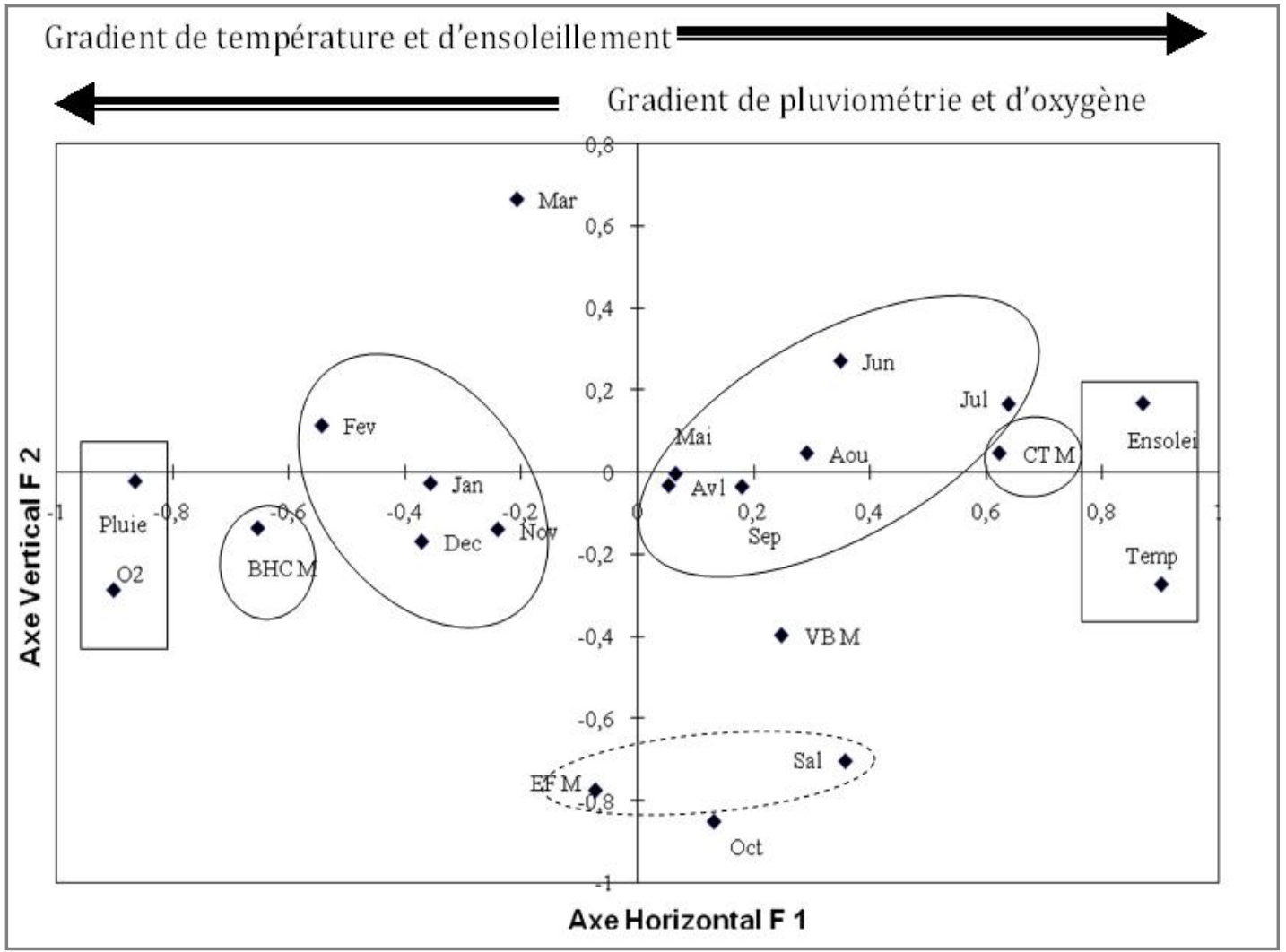

Figure 5. Analyse en composante principale des différentes facteurs abiotiques, et des charges en différents types bactériens dans les moules (Temp : température; Sal : salinité; Ensolei : Ensoleillement; Pluie : Pluviométrie; $\mathrm{O}_{2}$ : taux en oxygène dissout; $\mathrm{BHC} \mathrm{E}$ : bactéries hétérotrophes cultivables; CT E : coliformes totaux; EF E : entérocoques fécaux; VB E : Vibrionaceae).

Principal component analyses including the abiotic factors and charges of the different bacterial populations in the mussels. (Temp: temperature; Sal: salinity; Ensolei: sunshine; Pluie: rainfall; O2: the rate of oxygen; BHC E: heterotrophic cultivable bacteria; CT E: total coliforms; EF E: fecal enterococci; VB E: Vibrionaceae). 


\section{DISCUSSION}

L'espèce Mytilus galloprovincialis compte parmi les espèces cibles privilégiées pour l'aquaculture, notamment en Méditerranée. Cette espèce, qui se rencontre dans les eaux tempérées, chaudes ou froides, est considérée comme espèce sentinelle vu sa grande capacité de bioaccumulation (LE PENNEC et al., 2004). Cette espèce, introduite dans la lagune de Bizerte (Nord-Tunisie) depuis les années soixante, constitue le bivalve le plus exploité (DGPA, 1995).

Cependant, la lagune de Bizerte, comme tout écosystème côtier méditerranéen, a été marquée depuis les deux dernières décennies par un stress environnemental (grande variation haline), une baisse en biodiversité ainsi que de larges perturbations humaines induites par les rejets urbains organiques et industriels (VERLAQUE et al., 2007). Aussi, des périodes de mortalités par anoxie en période estivale se sont produites dans les stations mytilicoles dans la lagune (El HILI, 2005).

La présente étude a été menée en vue de déterminer l'influence des variations des facteurs du milieu sur la prolifération de différentes populations bactériennes dans un site mytilicole de la lagune de Bizerte.

Les résultats relatifs aux suivis des paramètres: $\mathrm{T}^{\circ} \mathrm{C}, \mathrm{O}_{2}$, $\mathrm{pH}$, salinité et pluviométrie ont montré que l'oxygène dissous, la température de l'eau, la salinité ainsi que la pluviométrie sont les facteurs les plus influents. Les taux en oxygène dissous seraient en corrélation négative avec la température de l'eau, alors que la salinité mesurée est inversement liée à la pluviométrie et dépend des apports en eaux douces dans la lagune. Ces résultats sont en concordance avec ceux de BEJAOUI et al. (2005), DELLALI et al. (2000, 2001) et DRIDI et al. (2007).

Par ailleurs, les résultats relatifs aux dénombrements des populations bactériennes ont montré que les sédiments sont relativement plus chargés que l'eau. Ceci refléterait la survie de ces bactéries dans les sédiments, ce qui serait conséquent à de fortes concentrations en matière organique (DELLALI et al., 2000, 2001; ROZEN et BELKIN, 2001).

Dans une étude effectuée sur le même site mytilicole (ESSID et al., 2007), les auteurs ont expliqué l'augmentation des charges bactériennes sédimentaires sous les tables d'élevage de moules par la richesse en matières organiques issues des feces et des pseudo-fecces de moules.

Selon LALIBERTE et GRIMES (1982), les sédiments représentent un réservoir d'accumulation de différentes formes bactériennes. Leur effet protecteur pour les espèces de bactéries pathogènes, notamment Aeromonas salmonicida (espèce pathogène en aquaculture), a été souligné par WIKLUND (1995).
D’autre part, les présents résultats ont révélé que les moules présentent des charges bactériennes comparables à celles des sédiments. Selon BELLA et TAM (2000) et MARINO et al. (2005), les moules (très grands filtreurs) peuvent capter plus rapidement les bactéries (notamment les coliformes dans l'eau ou les entérobactéries) et les retenir pour de longues périodes.

Dans la lagune de Bizerte, la production mytilicole est sujette à une surveillance sanitaire, contrôlant l'écoulement sur les marchés locaux ou l'exportation. Aussi, un prétraitement (d'épuration pour abattement des charges bactériennes) est effectué au préalable de leur commercialisation selon les normes CEE (NF ISO 6887-3, V 08-010-3, janvier 2004).

Les présents résultats ont révélé que les charges des bactéries fécales sont très importantes en saison hivernale, ce qui serait dû aux apports de rejets urbains drainés par les fortes pluies. Ainsi, nous avons constaté que les taux élevés en ces charges pendant le mois de janvier seraient associés avec l'augmentation pluviométrique, indiquant un apport certain d'eaux continentales. Ces observations sont confirmées par l'étude ACP appliquée à la colonne d'eau indiquant que les $\mathrm{BHC}$ et les EF seraient corrélés à la pluviométrie.

Cet effet d'apports continentaux sur la densité bactérienne a été bien mis en évidence dans d'autres sites conchylicoles par ASLAN-YILMAZ et al. (2004); CASTANEDA CHAVEZ et al. (2005); CROWTHER et al. (2001); DONNISSON et ROSS (1999); LIZARRAGA-PARTIDA et CARDENAS (1996) et SHIBATA et al. (2004). Aussi, LA ROSA et al. (2001) ont signalé la concomitance entre l'augmentation en taux de bactéries fécales dans les eaux d'une station mytilicole avec la période pluvieuse (septembre et janvier). Cette augmentation a été bien corrélée au lessivage sur les berges de la lagune.

Cependant, la survie de ces types de bactéries serait sous l'influence de la température (ROZEN et BELKIN, 2001). Dans le cas de la présente étude nous avons révélé une corrélation négative entre la température et les entérocoques fécaux.

Des résultats similaires ont été obtenus par ASLANYILMAZ et al. (2004), CHANDRAN et HATHA (2005), DAVIES-COLLEY et al. (1994) et SINTON et al. (2002). Ces auteurs ont montré que l'augmentation en température pendant la saison estivale inactiverait les polluants fécaux. Les travaux de MENON et al. (2003) ont également montré que la diminution en charges bactériennes allochtone et autochtone est sous l'effet de la prédation suite à l'augmentation en température de l'eau favorisant la prolifération des protozoaires. En plus de la température, la salinité serait aussi influente sur la multiplication de ces polluants fécaux dans les eaux marines peu profondes (MILL et al., 2006). 
Les résultats relatifs aux Vibrionaceaes ont révélé que leurs charges sont plus importantes que celles des bactéries allochtones fécales pour tous les prélèvements analysés, et ceci, durant toute la période d'étude. Ces vibrios semblent être une composante de la microflore des moules (LICCIANO et al., 2007). Selon la présente étude, les charges en ces bactéries dans l'eau ainsi que dans les moules sont faibles en période pluvieuse (caractérisée par une baisse de la température), ce qui serait dû à l'effet de dilution dans la lagune par l'arrivée d'eaux continentales. Aussi, l'analyse ACP a montré que l'influence de la température sur les VB est plus apparente dans l'eau que dans les moules.

Ces résultats sont similaires à ceux de RANDA et al. (2004) qui ont trouvé une corrélation positive entre l'abondance en vibrios, la température et la salinité dans les eaux côtières. Aussi, CASTANEDA CHAVEZ et al. (2005) ont montré que l'abondance des Vibrio cholerae dans des échantillons d'huîtres (Crassostrea virginica) est corrélée significativement avec l'augmentation de la température.

Par ailleurs, STABILI et al., (2005) soulignent une corrélation positive entre les charges en Vibrionaceaes dans les moules et les sédiments et la température de l'eau $(\mathrm{r}=0,90$ et $r=0,95)$. Selon ces auteurs les moules sont des réservoirs pour ces types de bactéries.

D'autre part, nous avons obtenu une corrélation positive entre les charges en Vibrionaceaes et celles des bactéries totales hétérotrophes dans le sédiment $(\mathrm{r}=0,650)$. Ces résultats sont cohérents avec ceux de La ROSA et al., (2001) dans leur étude comparant l'effet de la matière organique due à la biodéposition sur la qualité de l'eau et du sédiment dans une station mytilicole méditerranéenne. Les charges en BHC et en VB ont été associées à la richesse en matière organique dans les sédiments (ESSID et al., 2007). Il apparaît ainsi que ce sont les moules qui concentrent les BHC et particulièrement les VB qui seront libérés par leurs matières fécales puis stockés dans les sédiments, ce qui suggère que ces bactéries sont de bons indicateurs quant à la richesse en matière organique. Dans une étude antérieure, LIZARRAGA-PARTIDA et CARDENAS (1996) ont aussi révélé une forte corrélation $(r=0,97)$ entre les charges en VB et en BHC dans des échantillons de moules (Mytilus galloprovincialis).

\section{CONCLUSION}

La moule (Mitilus galloprovincialis) dans la lagune de Bizerte est soumise à un apport anthropique important, reflétant des charges bactériennes souvent comparables à celles des sédiments. Ces apports sont le plus souvent sous influence de la pluviométrie ainsi que la température et l'oxygène dessous.
Les différentes corrélations établies entre les charges en différents groupes bactériens et les facteurs du milieu ont révélé que les charges importantes en bactéries allochtones sont en relation avec les apports des rejets en période de forte pluviométrie (hiver et automne). Ces apports hydriques continentaux constituent une source de pollution fécale dans la lagune.

La température contrôle significativement la densité des vibrios, notamment dans les moules où l'élévation en charges de ces bactéries serait à l'origine de cas de mortalités estivales survenues dans le site mytilicole pendant la période d'étude. Il semble ainsi impératif de procéder au contrôle de risque de contamination humaine par ces vibrios, notamment en période estivale (période caractérisée par l'augmentation en consommation de ces moules).

Les sédiments qui constituent des réservoirs potentiels pour ces types de bactéries sont également à contrôler au même degré que les moules.

\section{REMERCIEMENTS}

Nous remercions M. Hosni Mahjoub ainsi que tout le personnel de la station mytilicole de Menzel Jemil (Bizerte) pour leur assistance technique durant la période de travail.

\section{RÉFÉRENCES BIBLIOGRAPHIQUES}

ASLAN-YILMAZ A., E. OKUS et S. ÖVEZ (2004). Bacteriological indicators of anthropogenic impact prior to and during the recovery of water quality in an extremely polluted estuary, Golden Horn, Turkey, Mar. Pollut. Bull., 49, 951-958.

BABARRO J. et A. DE ZWAAN (2002). Influence of abiotic factors on bacterial proliferation and anoxic survival of the sea mussel Mytillus edulis L., J. Exp. Mar. Biol. Ecol., 273, 33-49.

BEJAOUI B., R. BEN CHARRADA, M. MOUSSA, R. BEN HAMADOU, A. HARZALLAH et A. CHAPELLE (2005). Caractérisation hivernale de la lagune de Bizerte. Bull. Inst. Natl. Sci. Technol. Mer, 32, 79-91.

BELLA S. W. et T.Y. TAM (2000). Natural depuration of shellfish for human consumption: a note of caution. Water Res., 34, 1401-1406. 
BEN MAHMOUD I. (2003). Influence d'une contamination bactérienne sur le cycle biologique de Mytilus galloprovinvialis (Lmk) dans la lagune de Bizerte. Diplôme d'Etudes Approfondies, Institut National Agronomique de Tunis, $118 \mathrm{p}$.

BLANCHET H., N. RAYMOND, X. DE MONTAUDOUIN, M. CAPDEPUY et G. BACHELET (2003). Effects of digenean trematodes and heterotrophic bacteria on mortality and burying capability of the common cockle Cerastoderma edule (L.). J. Exp. Mar. Biol. Ecol. 293, 89-105.

BOUAMAMA K. (2001). Mytilus galloprovinvialis de la lagune de Bizerte: population bactérienne et biomarqueurs non spécifiques. Diplôme d'Etudes Approfondies en Ecologie Générale, Faculté des Sciences de Tunis, 99 p.

CASTANEDA CHAVEZM., V.P. SEDAS, E. O. BORUNDA et F. L. REYNOSO (2005). Influence of water temperature and salinity on seasonal occurrences of Vibrio cholerae and enteric bacteria in oyster-producing areas of Veracruz, Mexico. Mar. Pollut. Bull., 50, 1641-1648.

CAVALLO R. A. et A. STABILI (2002). Presence of vibrio in seawater and Mytilus galloprovinvialis (Lam) from the mar piccolo of Taranto (Ionian Sea). Water Res., 36, 3719-3726.

CHANDRAN A. et M. HATHA (2005). Relative survival of Escherichia coli and Salmonella typhimurium in tropical estuary. Water Res., 39, 1397-1403.

CHARMASSON S., E BARKER, A. PRUCHON et H. THÉBAULT (1999). Long-term variations of man-made radionuclide concentrations in a bio-indicator Mytilus galloprovinvialis from the French Mediterranean coast. Science Total Environ., 237, 93-103.

CHINA B., M. DE SCHAETZEN et G. DAUBE (2003). Les mollusques bivalves, des aliments dangereux. Ann. Med. Vet., 147, 413- 422.

CROWTHER J., D. KAY et M. D. WRYER (2001). Relationship between microbial water quality and environmental conditions in coastal recreational waters: the Fylde coast, UK. Water Res., 35, 4029-4038.

CUSSON M., R. TREMBLAY, G. DAIGLE et M. ROUSSY (2005). Modeling the depuration potential of blue mussels (Mytilus spp) in response to thermal shock. Aquacult., 250, 183-193.
DAVIES-COLLEY R. J., R. G. BELL et A. M. DONNISON

(1994). Sunlight inactivation of enterococci and fecal coliforms in sewage effluent diluted in seawater. Appl. Environ. Microbiol., 60, 2049-2058.

DELLALI M., M. EL BOUR et P. AISSA (2000). Évaluation de la pollution bactérienne dans la lagune de Bizerte: résultats préliminaires. J. Rech. Oceanogr., 26, 18-28.

DELLALI M., M. ELBOUR et P. AISSA (2001). Caractérisation des populations bactériennes de Vibrionaceaes de la lagune de Bizerte (Tunisie). J. Eur. Hydrol., 31, 91-103.

DPGA (DIRECTION GÉNÉRALE DE LA PÊCHE ET DE L'AQUACUlTURE) (1995). Plan Directeur de l'Aquaculture en Tunisie. Programme des Nations-Unies pour le Développement (PNUD), Ministère de l'Agriculture, République Tunisienne, $179 \mathrm{p}$.

DONNISON A.M. et C.M. ROSS (1999). Animal and human faecal pollution in New Zealand rivers, N. Z. J. Mar. Freshwater Res., 33, 119-128.

DRIDI S., M. ROMDHANE et M. ELCAFSI (2007). Seasonal variation in weight and biochemical of the Pacific oyster, Crassostrea gigas in relation to the gametogenic cycle and environmental conditions of the Bizerte lagoon, Tunisia. Aquacult., 263, 238-248.

EL BOUR M., H. ATTIA El HILLI, R. MRAOUNA et W. AYARI (2001). Bacterial study of mesophillic Aeromonads distribution in shellfish. Proceeding of the Fifth International Conference on the Mediterranean Coastal Environment, MEDCOAST 01, 23-27 octobre 2001, Hammamet, Tunisie, pp. 557-565.

EL HILI H. (2005). Rapport de mortalité de moules (Mytilus galloprovincialis) dans la lagune de Bizerte. Institut National des Sciences et Technologies de la Mer, Tunisie, rapport $\mathrm{n}^{\circ} 1319,20 \mathrm{p}$.

ESSID N., E. MAHMOUDI, F. BOUFAHJA, M. DELLALI, H. BEYREM et P. AISSA (2007). Impact des pseudo-feces de moules sur les densités des bactéries hétérotrophes dans le secteur mytilicole de la lagune de Bizerte (Tunisie). Rev. Sci. Eau, 20, 383-392.

GOURMELON M., M. P. MONTET, S. LOZACH, C. LE MENNEC, M. POMMEPUY, L. BEUTIN et C. VERNOZY-ROZAND (2006). First isolation of Shiga toxin $1 \mathrm{~d}$ producing Escherichia coli variant strains in 
shellfish from coastal areas in France. J. Appl. Microbiol., $100,85-97$.

KHESSIBA A., M. ROMÉO et P. AISSA (2005). Effects of some environmental parameters on catalase activity measured in the mussels (Mytilus galloprovinvialis) exposed to lindane. Environ. Pollut., 133, 275-281.

LALIBERTE P. et D. GRIMES (1982). Survival of Escherichia coli in lake bottom sediment. Appl. Environ. Microbial., 43, 623-628.

LA ROSA T., S. MIRTO, A. MARINO, V. ALONZO, T. L. MAUGERI et A. MAZZOLA (2001). Heterotrophic bacteria community and pollution indicators of musselfarm impact in the gulf of Gaeta (Tyrrhenian Sea). Mar. Environ Res., 52, 301-321.

LE PENNEC M., G. LE PENNEC, N. ALOUI-BEJAOUI et B. MÉTIVIER (2004). Les mytilidés et l'eau. Bull. Soc. Zoo. Fr., 129, 393-406.

LICCIANO M., L. STABILI, A. GIANGRANDE et R. A. CAVALLO (2007). Bacterial accumulation by Branchiomma lutuosum (Annelida: Polychaeta): A tool for biomonitoring marine systems and restoring polluted waters. Mar. Env. Res., 63, 291-302.

LIZARRAGA-PARTIDA M.L. ET G.V. CARDENAS (1996). Influence of water circulation on marine and fecal bacteria in a mussel-growing area. Mar. Pollut. Bull., 32, 196-201.

MARINO A., L. LOMBARDO, C. FIORENTINO, B. ORLANDELLA, L. MONTICELLI, A. NOSTRO et V. ALONZO (2005). Uptake of Escherichia coli, Vibrio cholerae non-O1 and Enterococcus durans by, and depuration of mussels (Mytillus galloprovincialis). Int. J. Food Microbiol., 99, 281-286.

MEDHIOUB M.N. (1999). La conchyliculture dans le lac de Monastir : Elevage de la moule commune, de l'huître creuse et de la palourde méditerranéenne. Institut National Scientifique et Technique d'Océanographie (INSTOP), Publication Trimestrielle ( $\left.\mathrm{n}^{\circ} 2\right)$. République Tunisienne, $22 \mathrm{p}$.

MENON P., G. BILLEN et P. SERVAIS (2003). Mortality rates of autochthonous and fecal bacteria in natural aquatic ecosystems. Water Res., 37, 4151-4158.

MILL A., T. SCHLACHER et M. KATOULI (2006). Tidal and longitudinal variation of fecal indicator bacteria in an estuarine creek in south-east Queensland, Australia. Mar. Pollut. Bull., 52, 881-891.

PAMPANIN D., I. MARANGON, E. VOLPATO, G. CAMPESAN et C. NASCI (2005). Stress biomarkers and alkali-labile phosphate level in mussels (Mytilus galloprovinvialis) collected in the urban area of Venice (Venice lagoon, Italy). Environ. Pollut., 136, 103-107.

RANDA M.A., M.F. POLZ et E. LIM (2004). Effect of temperature and salinity on Vibrio vulnificus population dynamics as assessed by quantitative PCR. Appl. Environ. Microbiol., 70, 5469-5476.

RIISGARD H., C. KITTNER et D. SEERUP (2003). Regulation of opening state and filtration rate in filterfeeding bivalves (Cardium edule, Mytillus edulis, Mya arenaria) in response to low algal concentration. J. Exp. Mar. Biol. Ecol., 284, 105-127.

ROZEN Z. et S. BELKIN (2001). Survival of enteric bacteria. FEMS Microbiol. Rev., 25, 513-529.

SHIBATA T., H.M. SOLO-GABRIEL, L.E. FLEMING et S. ELMIR (2004). Monitoring marine recreational water quality using multiple microbial indicators in an urban tropical environment. Water Res., 38, 3119-3131.

SINTON L.W., C. HALL, P.A. LYNCH et R.J. DAVIESCOLLEY (2002). Sunlight inactivation of fecal indicator bacteria and bacteriophages from waste stabilization pond effluent in fresh and saline water. Appl. Environ. Microbiol,. 68, 1122-1131.

STABILI L., M. IMMACOLATA et R.A. CAVALLO (2005). Mytilus galloprovincialis filter feeding on the bacterial community in a Mediterranean coastal area (Northern Ionian Sea, Italy). Water Res., 39, 469-477.

VERLAQUE M., C-F BOUDOURESQUE et F. MINEUR (2007). Oyster transfers as a vector for marine species introductions: a realistic approach based on the macrophytes. Dans: Impact of Mariculture on Coastal Ecosystem. D. BRIAND (Éditeur), 21-24 février, Lisboa, Portugal, CIESM Publications 32, 39-47.

WAECHTER M., F. LE ROUX, J.L. NICOLAS, E. MARISSAL et F. BERTHE (2002). Caractérisation de bactéries pathogènes de naissains d'huîtres creuses (Crassostrea gigas). C. R. Biol., 325, 231-238.

WIKLUND T. (1995). Survival of atypical Aeromonas salmonicida in water and sediment microcosms of different salinities and temperature. Dis Aquat. Org., 21, 137-143. 
YAM W.C., C.Y. CHAN, S.W. HO, T. TAM, C. KUEH et T. LEE (1999). Abundance of clinical enteric bacterial pathogens in coastal waters and shellfish. Water Res., 34, 51-56. 